\title{
Mass-to-Energy Conversion, the Astrophysical Mechanism
}

\author{
Conrad Ranzan \\ Astrophysics Deptarment, DSSU Research, Niagara Falls, Canada \\ Email: Ranzan@cellularuniverse.org
}

How to cite this paper: Ranzan, C. (2019) Mass-to-Energy Conversion, the Astrophysical Mechanism. Journal of High Energy Physics, Gravitation and Cosmology, 5, 520-551.

https://doi.org/10.4236/jhepgc.2019.52030

Received: March 14, 2019

Accepted: April 26, 2019

Published: April 29, 2019

Copyright (c) 2019 by author(s) and Scientific Research Publishing Inc. This work is licensed under the Creative Commons Attribution International License (CC BY 4.0).

http://creativecommons.org/licenses/by/4.0/

(c) (i) Open Access

\begin{abstract}
A new interpretation of the relativistic equation relating total-, momentum-, and mass-energies is presented. With the aid of the familiar energy-relationship triangle, old and new interpretations are compared. And the key difference is emphasized-apparent relativity versus intrinsic relativity. Mass-to-energy conversion is then brought about by adopting a three-part strategy: 1) Make the motion relative to the universal space medium. This allows the introduction of the concept of intrinsic energy (total, kinetic, and mass energies) as counterpart to the apparent version. 2) Recognize that a particle's mass property diminishes with increase in speed. This means introducing the concept of intrinsic mass (which varies with intrinsic speed). 3) Impose a change in the particle's gravitational environment. Instead of applying an electromagnetic accelerating force or energy in order to alter the particle's total energy, there will simply be an environmental change. Thus, it is shown how to use relativity equations and relativistic motion-in a way that exploits the distinction between apparent and innate levels of reality-to explain the mass-to-energy-conversion mechanism. Moreover, the mechanism explains the 100-percent conversion of mass to energy; which, in turn, leads to an explanation of the mechanism driving astrophysical jets.
\end{abstract}

\section{Keywords}

Relativistic Mass Energy, Kinetic Energy, Momentum Energy, Total Energy, Mass-Energy Conversion, Intrinsic Mass, Terminal Neutron Star, Energy Emission Mechanism, Astrophysical Jets, DSSU Theory

\section{Introduction}

The second year of the third millennium saw the conceptual origination of the 5 fundamental processes that drive the universe, that sustain its perpetual existence, that make the world what it is. In that year, 2002, the ruling framework of 
the cosmos was uncovered. The present article details the $13^{\text {th }}$ major discovery to emerge from the theory built on the foundation of those original processes. What follows details the discovery of the total conversion of mass to energy-without the involvement of antimatter.

The purpose of the presentation is threefold: The first is to present the mathematical underpinning of the mass-to-energy conversion. The second is to detail the physical aspects of the conversion mechanism. The third is to examine the role that mass conversion plays in powering a major astrophysical phenomenon.

Within the following pages, it will be shown how to use relativity equations to explain mass-to-energy conversion, which, in turn, contributes in a major way to the explanation of the mechanism driving astrophysical jets.

\section{The Energy Triangle}

For an understanding of the motion of objects and particles at a fundamental level, several forms of energy must be taken into account, namely the kinetic energy and the energy associated with momentum, as well as the total energy and the mass energy. The mathematical expressions for these energies will be developed and shown to be valid (if properly interpreted) for all speeds, from zero to lightspeed. Naturally, the four energies are mathematically related; in fact, they are related in such a way that they can be configured into a most useful energy triangle.

\subsection{Momentum}

First, consider the energy associated with momentum. The problem with the classical momentum expression is connected to the velocity $v$. Velocity is a measurement, by definition, that depends on clock time. The faster you travel, the slower your accompanying time-keeping device (clock) will run. Joseph Hafele and Richard Keating, back in 1977, performed the experiments that proved this to be true.

What this means for a relativistically moving particle is that it will cover a significantly greater distance during a time interval (say one second of its "slow-clock" time) compared to a one second interval recorded on a stationary clock. When the stationary observers measure the particle's speed (and hence, its momentum) they, of course, must use their own clock time. The difference in the clock times must be incorporated into the momentum equation.

The classical expression for the magnitude of the momentum of a particle moving with constant speed in the $x$-direction is

$$
p=m v=m \frac{\Delta x}{\Delta t}
$$

in which $\Delta x$ is the distance covered in time $\Delta t$.

As a first step to finding the relativistic expression for momentum, its definition is changed so that the velocity incorporates moving-clock time. That is, $v$ is 
now expressed as $\Delta x / \Delta t_{0}$, so that

$$
p=m \frac{\Delta x}{\Delta t_{0}}
$$

As before, $\Delta x$ is the distance travelled by a particle as viewed by an observer watching that particle. However, $\Delta t_{0}$ is the time required to traverse that distance, measured not by the observer watching the moving particle but by a hypothetical observer moving with the particle. This $\Delta t_{0}$ is the particles "proper" clock-time. A simple expansion of (2) gives [1]

$$
p=m \frac{\Delta x}{\Delta t_{0}}=m \frac{\Delta x}{\Delta t} \frac{\Delta t}{\Delta t_{0}} .
$$

The middle term $(\Delta x / \Delta t)$ is just the particle's velocity $v$; and $\left(\Delta t / \Delta t_{0}\right)$ is the velocity-dependent clock-time ratio $1 / \sqrt{1-(v / c)^{2}}$ or $\gamma[2]$. With these substitutions, the general definition for momentum, in vector form, is

$$
\boldsymbol{p}=m \boldsymbol{v} \cdot \text { (momentum) }
$$

The thing to note is that, as the velocity approaches lightspeed $c$, the predicted magnitude of momentum tends to infinity. The true meaning of this infinity will be explained later, in Section 3.

\subsection{Total Energy}

The total energy $E$ of a free body or particle is defined as [3]

$$
E=m c^{2} \gamma
$$

where $\gamma$ is again the velocity-dependent clock-time ratio. Now, since the total energy consists of two parts, the rest energy $E_{0}$ and the kinetic energy $E_{\text {kin, }}$, the full expression may be stated as

$$
E=E_{0}+E_{\text {kin }}=m c^{2} \gamma .
$$

There is nothing to prove here, since this is assumed to be true by definition. (Just be aware of the potential infinity).

Now, there is a simple way to bring together (into one equation) the total energy, rest energy and motion energy. It is a matter of algebraically combining Equation (4) and Equation (5) to obtain

$$
E^{2}=\left(m c^{2}\right)^{2}+(p c)^{2} .
$$

The proof is given in the Appendix.

Another useful equation is one that relates total energy $E$ and momentum $p$. It is derived by combining Equation (4), $\boldsymbol{p}=m \boldsymbol{v} \gamma$, and Equation (5), $E=m c^{2} \gamma$, as follows:

From Equation (5), is obtained $m=\frac{E}{c^{2} \gamma}$. Then, by substitution, Equation (4) becomes $\boldsymbol{p}=m \boldsymbol{v} \gamma=\boldsymbol{v} \frac{E}{c^{2} \gamma} \gamma$, or simply, 


$$
\boldsymbol{p}=\boldsymbol{v} \frac{E}{c^{2}},
$$

an expression for momentum in terms of velocity and total energy.

\subsection{Rest Energy}

The rest energy of a body or particle is found with Equations (6)-(8). When the velocity is zero, then momentum $\boldsymbol{p}$ will equal zero. And with $\boldsymbol{p}=0$, the expanded Equation (7) changes as follows:

$$
\begin{gathered}
E^{2}=\left(E_{0}+E_{\mathrm{kin}}\right)^{2}=\left(m c^{2}\right)^{2}+(\boldsymbol{p} c)^{2} \\
\left(E_{0}+0\right)^{2}=\left(m c^{2}\right)^{2}+0 ; \\
E_{0}=m c^{2}
\end{gathered}
$$

Notice that this equation works for all particles and all velocities, with the understanding that anything with (or of ) mass cannot travel at lightspeed. For any particular particle, the rest energy $E_{0}$ does not vary-it is an innate property. For particles with mass, $m$ is constant and $E_{0}>0$. As an example, the rest energy of an electron is 0.511 Million electron Volts $(\mathrm{MeV})$. While for a particle with no mass, $m$, obviously, is zero and $E_{0}=0$. The photon is the patent example; it has no mass and since it does not exist in a rest state, the photon's rest mass equals zero. Equation (9) is consistent with the reality in both cases.

Note, however, what happens with the commonly misapplied expression, $E=m c^{2} \quad$ (Here the symbol for total energy $E$ is used instead of the correct one for rest energy). If energy of a particle varies, as happens, then the mass $m$ must, with the use of this form of the equation, also vary (The speed of light $c$, of course, always remains constant). With the terminology of this expression, a clear identification of a particle's rest energy is lost. Moreover, it conveys the implication that a photon possesses inertial mass! For the massless photon, this expression predicts $m=E_{\gamma} / c^{2}$, where $E_{\gamma}$ is the energy of the photon-energy that cannot be zero. Thus, it wrongly attributes the photon with a mass value.

In other words, $E_{0}=m c^{2}$ is the preferred and logically more consistent expression for rest energy.

\subsection{Energy Triangle}

The energy formulations can be combined in a graphic way. Here is the bare-bones version of the energy equation-based on Equation (6) and Equation (7) from above:

$$
(\text { Total energy })^{2}=(\text { Rest energy })^{2}+(\text { Momentum energy })^{2} .
$$

Since it has the basic Pythagorean form, it can be configured as a right-angled triangle (Figure 1).

The range of validity is checked as follows.

Low-speed test. What is the prediction for non-relativistic speeds?

From Equation (6) and Equation (7), 


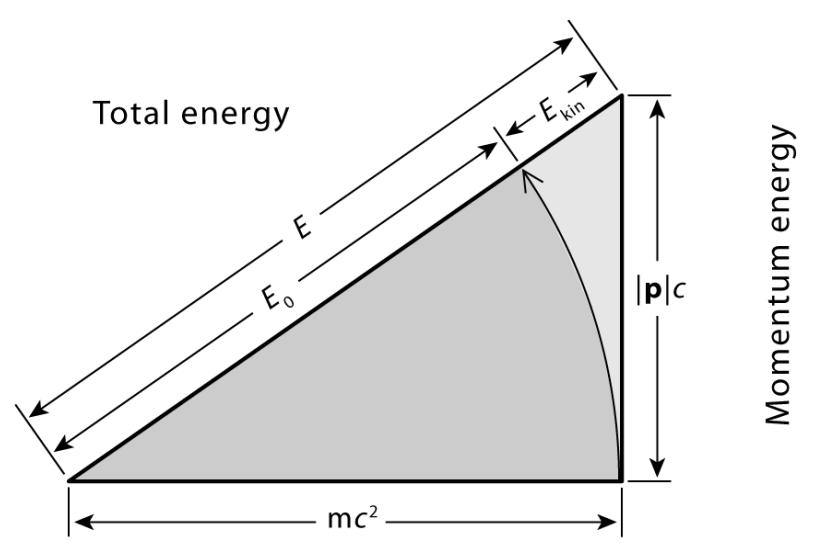

Rest energy

Figure 1. The energy triangle embodies the Pythagorean relationship among the energy components that may be associated with bodies or particles; and most relevantly to elementary particles-be they mass or massless, be they stationary or moving at the speed of light.

$$
\begin{aligned}
E & =E_{0}+E_{\mathrm{kin}}=\sqrt{\left(m c^{2}\right)^{2}+(\boldsymbol{p} c)^{2}} \\
& =\left(m c^{2}\right)\left(1+\frac{(\boldsymbol{p} c)^{2}}{\left(m c^{2}\right)^{2}}\right)^{1 / 2} \\
& =\left(m c^{2}\right)\left(1+\frac{\boldsymbol{p}^{2}}{(m c)^{2}}\right)^{1 / 2}
\end{aligned}
$$

For low speed, $\frac{\boldsymbol{p}^{2}}{(m c)^{2}} \ll 1$. Then, with the application of the binomial expansion theorem,

$$
E_{0}+E_{\text {kin }} \approx\left(m c^{2}\right)\left(1+\frac{1}{2} \frac{\boldsymbol{p}^{2}}{(m c)^{2}}+\cdots\right)=m c^{2}+\frac{\boldsymbol{p}^{2}}{2 m}+\cdots
$$

which gives the low-speed kinetic energy as

$$
E_{\mathrm{kin}} \approx \frac{\boldsymbol{p}^{2}}{2 m}=\frac{1}{2} m v^{2} \text {. }
$$

The prediction is in agreement with the Newtonian version of kinetic energy.

Mass test: What do the equations predict if the particle has zero mass?

Equation (7) and Equation (8), $E^{2}=\left(m c^{2}\right)^{2}+(\boldsymbol{p c})^{2}$ and $\boldsymbol{p}=\boldsymbol{v} \frac{E}{c^{2}}$, can be used to prove that when $m=0$ the velocity of the particle is $c$ in any reference system. There is no rest frame for such "bodies". They have no rest energy; their total energy is purely kinetic [4].

Ultimate-speed test: What is predicted for a particle with lightspeed? It is just the converse of the above test, using Equation (7) and Equation (8). If a particle has velocity $c$, then its mass evaluates to zero-as must be the case for pure energy 
radiation.

Energy triangle applicability:

When applied to a stationary mass, the energy triangle collapses into its base (Figure 2(a)).

When applied to a moving mass, all the energy components are present (Figure 2(b)).

When applied to a pure energy particle, the energy triangle squeezes into its height (Figure 2(c)). In other words, when mass equals zero, $E_{\text {kin }}=p c$ and the base of the triangle vanishes.

\section{Traditional Manipulation of the Energy Triangle}

\subsection{Mass Deemed Constant}

The traditional approach in applying the relativistic energy equations (represented by the energy triangle) is to treat the mass energy as a fixed quantity. For example, the base of the triangle for a speeding proton always equals $938 \mathrm{MeV}$ (or $1.50 \times 10^{-10}$ joules). This now-well-established stratagem among physicists is to elevate the status of mass and keep it constant-regardless of the state of motion. The modern view is that there is no distinction between rest mass and relativistic mass. True enough, Einstein and his followers for a good number of years claimed that mass increases with speed-it was, after all, part of the original special relativity theory. Sir Arthur S. Eddington was among those who falsely believed that mass increases with speed. But, as with several of his other early viewpoints, Einstein reconsidered and changed his stance. In a private letter in 1948 , he made it clear that he had abandoned the idea, stating,

"It is not good to introduce the concept of the mass $M=m / \sqrt{1-v^{2} / c^{2}}$ of a moving body for which no clear definition can be given. It is better to introduce no other mass concept than the "rest mass" $m$. Instead of introducing $M$, it is

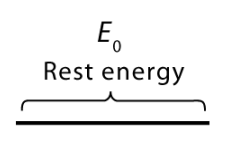

Stationary

mass particle

(a)

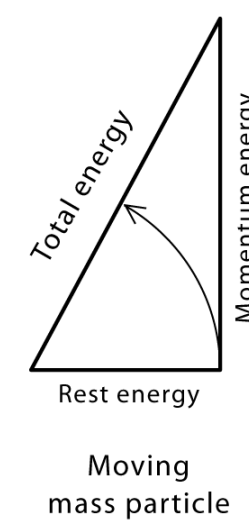

(b)

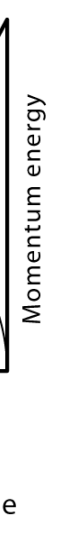

Energy particle
(e.g., photon, neutrino)
Energy particle
g., photon, neutrino)

(c)

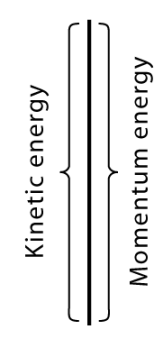

Figure 2. Energy "triangle" accommodates both mass and massless particles. (a) A free stationary particle has only rest energy; (b) a moving mass particle has a full complement of energy components; (c) energy particles have no rest energy, thus the two other sides of the triangle come together so that the two energies of motion are equal. 
better to mention the expression for the momentum and energy of a body in motion"-A. Einstein, in letter to Lincoln Barnett [4].

The question is, How can the energy triangle (and the relativity equations) be used to represent the conversion of mass to energy? What must take place in the physical world? ... The most essential requirement is that the mass particle must attain the speed of light. Only then, will the mass be in the form of pure energy. But how is the speed to be attained? Yes, there is an innate problem, a theoretically imposed condition, a textbook mantra.

Nevertheless, imagine just such an attempt. A graphic demonstration (Figure 3) shows what happens as a charged particle is driven step-by-step to ever higher speeds using an imagined unlimited supply of external energy. As the force is applied, the speed increases, the kinetic energy (and hence total energy) and momentum energy increase. The mass energy, by the conventional rules of relativistic physics, remains invariant. The vertical side grows in length, so does the hypotenuse, while the base representing the mass remains unchanged. As the speed approaches that of light the energy input tends to infinity.

As the hydrogen atom's speed approaches that of light, its kinetic energy grows dramatically. When the speed is $99.999 \%$ of lightspeed, it has the equivalent kinetic energy of 1 grain of table salt dropped from a height of 1 centimeter. At $99.999,999,999,99 \%$ of lightspeed, it has the kinetic energy of 1 buckshot pellet dropped from a height of 1 centimeter. And at 99.(+26 nines)\% of lightspeed, the hydrogen atom is predicted to have the equivalent kinetic energy of a motorcycle travelling 25 miles per hour! For the latter example, the energy of motion would be $7.1 \times 10^{13}$ times greater than the hydrogen's basic mass energy [5].

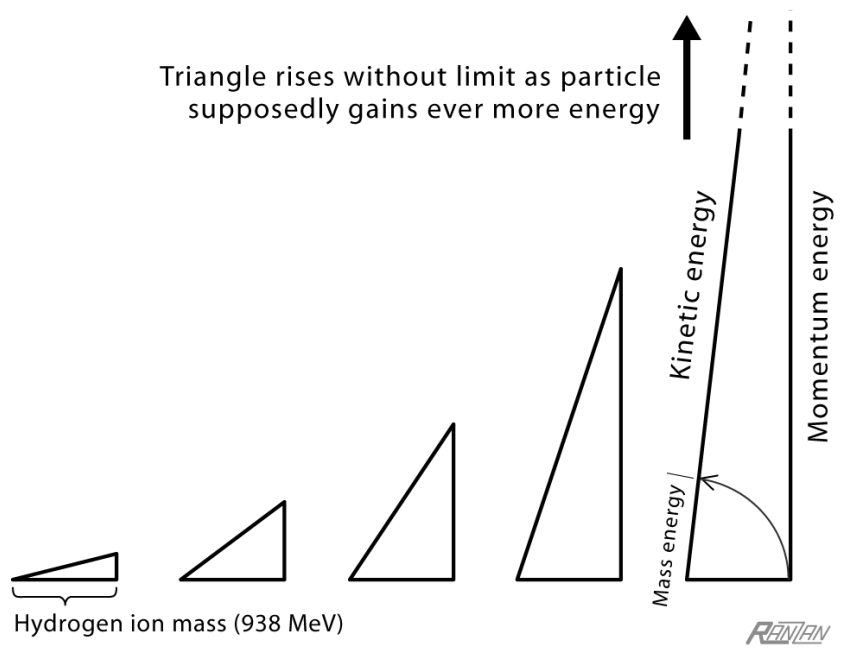

Figure 3. Conventional way of manipulating the energy triangle is to hold the base constant (in light of the assumption that mass is invariant) and then increase the triangle's height. Consider a hypothetical example for a hydrogen ion; electric field energy is applied in ever greater intensity-while the ion gains ever more energy of motion. There is no theoretical limit to this process, and as the energy grows without restriction, the speed approaches $c$. However, the particle never attains lightspeed and never becomes massless. It always retains its mass (Hydrogen mass is $1.67 \times 10^{-27}$ kilograms). 
It is doubtful that anyone believes that a mere proton, a stripped-down hydrogen atom, travelling very close to the speed of light, could actually possess the energy equivalence of a cruising motorcycle. Pause and think about it. Something else must be going on.

\subsection{Problems with the Traditional Approach}

Applied energy versus acquired energy: Granted, the equations don't lie; but how is it possible to seriously suggest that a hydrogen atom could, within the realm of physics, amass the energy of a cruising motorcycle!? ... It requires enormous faith in one simple assumption-the assumption that the energy, the electromagnetic field energy, applied to the charged particle is equal to the energy the particle actually acquires.

What about the likelihood that the energy expended in moving the hydrogen atom (or ion), is not necessarily the energy gained and possessed by it? Where the vast amount of applied energy goes, if not acquired by the particle, is not here important. The more realistic view is that the equations are representative of the external energy required to accelerate the particle/body to attain relativistic speed. It makes much more sense to say that no vast amount of applied energy could propel something to lightspeed, than it is to say that the particle's energy tends to infinity as its speed approaches lightspeed!

The equation-predicted energy for extreme motion may well be the applied energy; but it most certainly is not what is possessed by the speeding atom.

Apparent versus intrinsic energy of motion: The problem here is that conventional Physics draws no distinction between apparent and intrinsic forms of kinetic energy. And in the context of the mass-to-energy discussion, it means a failure to distinguish between the motion of conventional relativity and motion relative to the universal medium (motion with respect to aether).

This touches on what is one of the biggest shortcomings in all physics-the failure to draw distinctions among the three types of fundamental motions. They are as follows: motion through aether, motion with aether, and motion irrespective of aether (that is, ordinary relative motion). They require different interpretations of equations; and may lead to radically different results. A dramatic and generally unrecognized example is the total cancellation of the centrifugal effect. Without recognizing the difference between motion through aether and with aether, significant astronomical rotation cannot be understood; and has led to the invention of dark matter, a hypothetical substance that compounds the bafflement of astrophysicists. Another example, where aether-referenced motion is important, is revealed in the difference between apparent and intrinsic radiation energy. The wrong interpretation here has trapped proponents of the Sachs-Wolf effect-an hypothetical process that has recently been disproved [6]. What had been long thought by physicists to be an energy gain by photons during segments of the cosmic journey was actually an intrinsic energy loss!

The infinity problem: When the motion-energy equations are interpreted as 
in Figure 3, the energy tends to infinity. Equations that behave this way are a red flag. The underlying phenomenon that the equation represents is losing its connection to reality. The underlying theory is pushing beyond its legitimate domain. Such drift to infinity is the signal that some other interpretation needs to be explored.

Problem of the missing unifying theory: Conventional Physics lacks a unifying theory for mass and energy particles. And worse, some theorists adamantly maintain a strict distinction between the two, and hold that there is one conservation law for mass and another law for energy-thus, precluding the conversion of one to the other [7]. Without some fundamental connection between mass and energy, without something more than just mathematical symbolism, explaining their interconvertibility becomes an insurmountable task. Since the goal here is to explain mass-to-energy conversion, a unifying theory is essential.

A simple solution to this problem is already in the literature and will be put to use in the next Section.

The primacy-of-mass problem: The fundamental problem is that the mass is treated as invariant regardless of speed. No increase of mass with increase of motion, that is the standard view; but that, in itself, is not the problem. Unfortunately, the "invariant" view also precludes the possibility that the mass may actually (intrinsically) decrease with extreme motion. It turns out, as will be explained shortly, that just such a scenario in which a particle diminishes its mass-value as it approaches the speed of light resolves the mass-to-energy question.

Restrictive nature of relativity theory. There is a fundamental limitation built into relativity theory. Its motion and energy equations are rooted in electrodynamics and form an essential part of relativity theory. This connection is explicit in the title of Einstein's seminal 1905 paper (considered to be the foundation piece for modern relativity), "On the Electrodynamics of Moving Bodies", and imposes a profound barrier. The key to understanding this lies in the term "electrodynamics", which means there is force and/or energy involved. Always. (It is a key point, for an alternate barrier-free process does exist).

In general, the equations are applicable to all speeds (as demonstrated earlier). However, an energy divergence situation-a divergence to infinity-can arise and must be correctly interpreted. Most emphatically, it does not mean that a subluminal particle is precluded from attaining lightspeed. Not at all. It is only within Einstein's theory that the attainment of lightspeed is precluded. In that theory, the "infinity" is a fundamental restriction; it signals the fact that no electrodynamic force, no electromagnetic field, can ever drive a charged particle up to the full speed of light.

In the context of this paper's theme, the electrodynamic force cannot directly cause mass-to-energy conversion.

The relativity equations must be interpreted in the light of the fact of being rooted in the physics of electrodynamics. There is, however, another way-another interpretation. 


\section{Alternate Approach in Applying the Relativistic Energy Equation}

\subsection{Space Medium, Mass Diminishment, Gravity Influence}

The fact, then, is that no applied force can ever cause a mass particle to attain lightspeed and effect a mass-to-energy conversion. And it makes perfect sense. There is an intuitive way to understand the reason. It's a simple matter of a diminishing return on energy invested.

Consider the problem from this perspective. In particle accelerators, powerful magnetic fields are used to accelerate charged particles such as protons or electrons. Plain and simple, the force driving the particles is an electromagnetic field; the charged particles are being "pushed" by the photons of the electromagnetic field. It should be obvious that when the speed difference is large, when the lightspeed photons are interacting with stationary or slow-moving protons, the accelerating "push" will be greatest. It should be equally obvious that when the speed difference is small, the push-effect will be comparatively weaker. The push provided by the field will produce a smaller and smaller incremental change in the speed-a diminishing return on energy invested. Regardless of the intensity of the accelerating electromagnetic field, the closer the charged particles (protons or electrons) are driven to lightspeed, the more negligible will be the effect of the force experienced by the particles. In the theoretical limit, as the field force becomes infinitely great, the increase in speed becomes vanishingly small. Lightspeed can never be attained. Nevertheless, as will be demonstrated in a moment, it is not the fundamental equations (the energy equations) that stand in the way.

Einstein described the diminishing potency of the driving force this way:

" $A$ body at rest has mass but no kinetic energy, that is, energy of motion. A moving body has both mass and kinetic energy. It resists change of velocity more strongly than the resting body. It seems as though the kinetic energy of the moving body increases its resistance. If two bodies have the same rest mass, the one with the greater kinetic energy resists the action of an external force more strongly" [8].

The conventional approach, then, is a conundrum - a catch-22 situation. Mass converts to pure energy, provided it can be compelled to travel at lightspeed. But in order to attain lightspeed the mass must first convert to pure energy. Call it the dilemma of the unreachable speed.

In order to resolve the dilemma, three changes must be introduced.

One. Make the motion relative to the universal space medium. This allows the introduction of the concept of intrinsic energy (total, kinetic, and mass energies) as counterpart to the apparent version.

Two. Recognize that a particle's mass property diminishes with increase in speed. This means introducing the concept of intrinsic mass (which varies with intrinsic speed).

Three. Instead of applying an electromagnetic force or energy, there will 
simply be an environmental change-a change in the particle's gravitational environment.

The reasonableness of these changes will be discussed in a moment, but first, the specifics of the alternate approach. The strategy is to interpret the energy triangle in terms of intrinsic parameters. That is, first and foremost, the velocity of the particle/body is taken to be with respect to the universal space medium (henceforth, the aether). This intrinsic velocity $v_{\text {int }}$ also appears in the gamma factor-which now becomes the intrinsic gamma factor $\gamma_{\text {int }}$. And since mass decreases with speed (per introduced change number "Two"), it too becomes an intrinsic parameter. The new-approach energy triangle, appropriately labelled with the revised parameters, is shown in Figure 4.

The total energy equation is now expressed as

$$
E_{\text {int }}=E_{\text {int.mass }}+E_{\text {int.kin }} \text {. }
$$

And from Figure 4 triangle it is obvious that

$$
\begin{gathered}
E_{\text {int }}^{2}=\left(m_{\text {int }} c^{2}\right)^{2}+\left(\boldsymbol{p}_{\text {int }} c\right)^{2} ; \\
E_{\text {int }}=\sqrt{\left(m_{\text {int }} c^{2}\right)^{2}+\left(\boldsymbol{p}_{\text {int }} c\right)^{2} .}
\end{gathered}
$$

The important difference is that mass is now a function of aether-referenced motion and can vary from rest-mass-value $m_{0}$ all the way down to zero (Figure 5). In terms of the gamma factor,

$$
m_{\mathrm{int}}=\frac{m_{0}}{\gamma_{\mathrm{int}}} .
$$

And in terms of the velocity,

$$
m_{\mathrm{int}}=\sqrt{1-\left(v_{\mathrm{int}} / c\right)} m_{0} .
$$

As convention has it, $m_{0}$ is the rest mass and remains constant within the equation (i.e. $m_{0}$ is not a variable parameter).

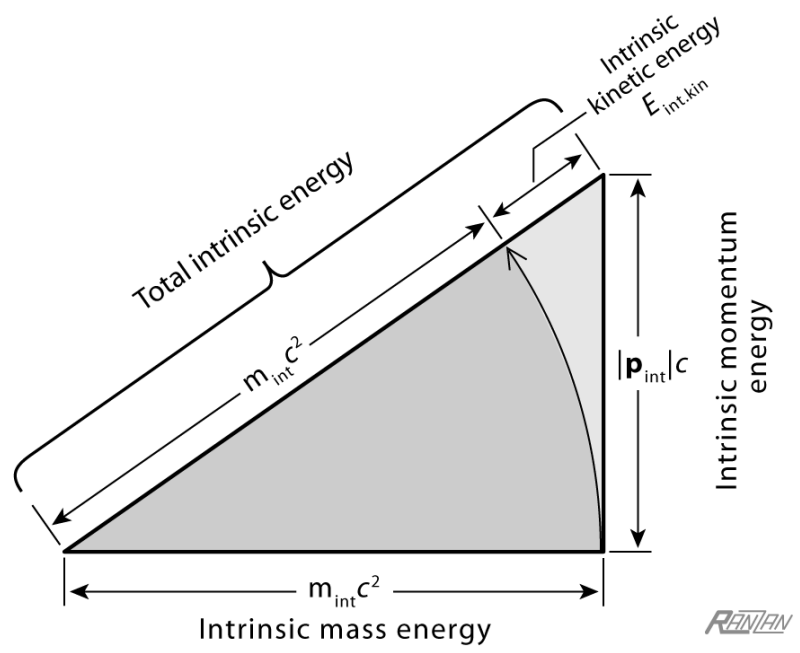

Figure 4. Energy triangle applicable to particles/objects for which velocity is referenced to the universal space medium. Intrinsic mass $m_{\text {int }}$ is defined as $\left(m_{0} / \gamma_{\text {int }}\right)$. And $\boldsymbol{p}_{\text {int }}$ is defined as $\left(m_{0} v_{\text {int }} / \gamma_{\text {int }}\right)$. 


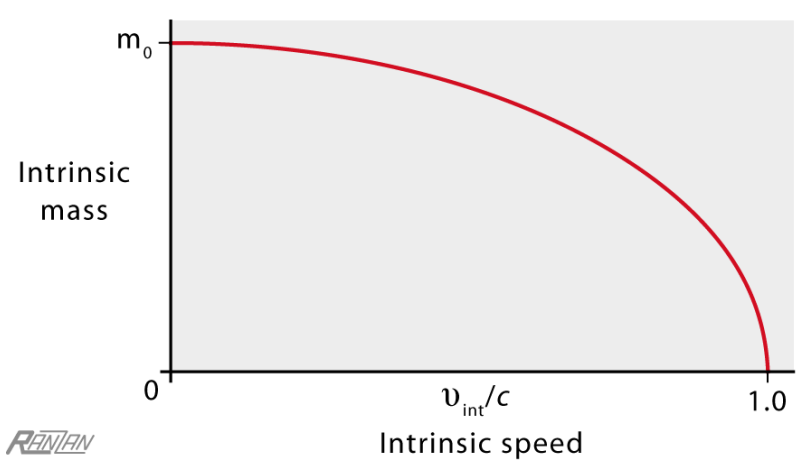

Figure 5. How mass varies with speed. Specifically, how it varies when the cause of the change in speed is not an applied force but, rather, a change in the particle or body's gravitational environment (Symbol $m_{0}$ is the rest mass and $v_{\text {int }}$ is the aether-referenced speed).

An expression for the kinetic energy follows directly from the energy triangle (Figure 4).

$$
E_{\text {int.kin }}=\sqrt{\left(m_{\mathrm{int}} c^{2}\right)^{2}+\left(\boldsymbol{p}_{\mathrm{int}} c\right)^{2}}-\left(m_{\mathrm{int}} c^{2}\right) .
$$

So, aether referencing has been adopted and mass variability has been introduced. Now for the third item, changing the speed without applying energy. This change comes with a most convenient opportunity.

Since no energy is to be applied to the mass, as only an alteration it its gravitational surroundings will take place, it is, therefore, reasonable to assume a strict adherence to the conservation of energy principle-starting with the mass energy and ending with the same amount, but in pure radiation form.

What it means in the schematic representation (Figure 6) of this mass-to-energy conversion is that the hypotenuse remains unchanged throughout the transformation. The total intrinsic energy magnitude remains constant.

The concept of mass decrease (diminishment with increasing speed) changes our understanding of the energy of motion. It leads to the radical difference between the kinetic energy of Figure 3 and that of Figure 6. Recall the textbook definition. The kinetic energy is said to be "the work required to accelerate the particle from rest to its observed speed". But what if no work is done, no force is applied? And there is not even a change of speed, at least not in the conventional sense. What then?

What if the particle is simply subjected to an unavoidable change in its gravitational situation? In such a case, a broader view of kinetic energy is necessary, one that incorporates a dual interpretation based on two kinds of motion-apparent and intrinsic. In order to cover all situations, there must be two fundamentally different interpretations of kinetic energy. See Table 1.

The differences are significant: Apparent energy versus intrinsic energy. Total energy is variant versus remains constant. Kinetic energy varies with external force/energy applied to object/particle versus its variance with the state of its intrinsic motion (or its gravitational environment). Mass invariance versus mass 


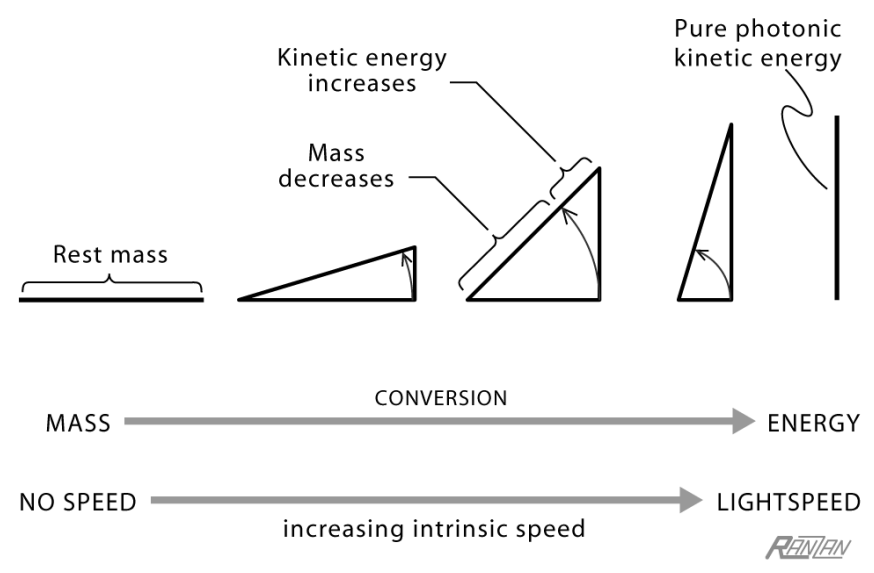

Figure 6. Schematic demonstration of mass-to-energy conversion (The hypotenuse is held constant as the base shrinks with increasing speed). Since no force is applied, under the condition described in the text, there is no reason to expect any change to the total energy (mass energy plus kinetic energy). Thus, the energy triangle's hypotenuse remains constant during the conversion. Conservation law is obeyed; with no loss or gain.

Table 1. Dual interpretation of kinetic energy. The energy of motion has two distinct interpretations-the conventional one based on apparent motion and the fundamental one based on intrinsic (aether-referenced) motion.

\begin{tabular}{lrl}
\hline & $\begin{array}{c}\text { Energy of Motion } \\
\text { KINETIC ENERGY }\end{array}$ \\
\hline \multicolumn{1}{c}{ Conventional view } & & \multicolumn{1}{c}{ Fundamental view } \\
\hline $\begin{array}{l}\text { - Relative motion between inertial } \\
\text { frames }\end{array}$ & MOTION & $\begin{array}{l}\text { - Relative motion between particle and } \\
\text { aether }\end{array}$ \\
- Equals work applied & DEFINED & - Defined only by intrinsic speed \\
- Observer dependent & OBSERVER & - Observer independent \\
- No upper limit (depends on & LIMIT & $\begin{array}{l}\text { - Has upper limit (when particle attains } \\
\text { force/energy applied) }\end{array}$ \\
- Precludes mass-to-energy conversion & CONVERSION - Involves mass-to-energy conversion \\
- Basic physics; particle physics & USEFULLNESS - Study of gravitational collapse \\
- APPARENT kinetic energy & TERMINOLOGY - INTRINSIC kinetic energy
\end{tabular}

decrease (with increasing speed). The asymptotic approach to speed $c$ versus the actual attainment of lightspeed.

Most importantly, the intrinsic interpretation is crucial for understanding the gravitational conversion of mass to pure energy.

\subsection{Justification Discussion}

Aether. The first change really needs no elaboration. Einstein [9] invoked the aether concept, Michelson and Morley [10] back in 1887 had detected an aether speed of 5 to 7.5 kilometers per second, Dayton Miller [11] during the 1920s confirmed its detection, and the De Witt experiment [12] requires its presence. No serious researcher in physics is willing to categorically deny the existence of a universal space medium. 
Non-constancy of mass. The reality of mass decrease with speed is compellingly argued by H. I. Epstein, in a 2009 Physics Essays article [13]. In the next section, the DSSU mechanism of mass reduction is clearly described.

Change "Three" invokes a force that is not a true force: Be reminded that the electromagnetic force is a true force (and according to DSSU theory, it is the universe's only fundamental force). Gravity, on the other hand, is not a true force; there are no force-carrier particles; gravity is simply an effect produced by the flow gradient of the aether. So, while an electromagnetic force when applied to a charged particle is expected to increase its energy, there is no reason to expect the mere presence of gravity to alter the energy of an at-rest particle/body. But note, under the new interpretation, it is the total energy that remains constant regardless of the gravity intensity. Not so for the mass energy.

As a fundamental point of distinction, the conventional view is that mass energy remains constant regardless of the gravity intensity. Place a 1 kilogram mass onto the surface of an earth-like planet, earth-size but with a much higher density, and the gravitational "pull" will be correspondingly much greater-but by the conventional interpretation the mass does not change, it will still be only 1 kilogram.

It turns out that when extreme gravity is the driver (with no electromagnetic force applied to the particle), the expectation is a clean mass-to-energy conversion.

In summary, the adoption of the three changes was justified, as was the reinterpretation of the original energy triangle.

\section{Mass and the Conversion Mechanism}

Demonstrating the mass-to-energy conversion using the energy triangle was quite straightforward; demonstrating the physical mechanism of the conversion is equally uncomplicated, if not more so.

In the early part of the $20^{\text {th }}$ century, physicists became aware of the deep connection between mass and radiation particles. One who expressed the connection most clearly was the eminent English physicist and astronomer James Hopwood Jeans (1877-1946). In his 1931 book, The Mysterious Universe, he championed the idea that mass is simply the manifestation of confined particles of radiation-the wave-energy particles of light. Quoting from the chapter, Matter and Radiation,

"... $[W]$ e may come fairly near to the truth if we think of matter and radiation as two kinds of waves-a kind which goes round and round in circles, and a kind which travels in straight lines. The latter waves of course travel with the velocity of light, ... this may express the whole difference between matter and radiation, matter being nothing but a sort of congealed radiation travelling at less than its normal speed" [14].

And within the same chapter, Jeans expands the idea to include matter conversion. 
"... [T] he tendency of modern physics is to resolve the whole material universe into waves. These waves are of two kinds. bottled-up waves, which we call matter, and unbottled waves, which we call radiation or light. The process of annihilation of matter is merely that of unbottling imprisoned wave-energy and setting it free to travel through space" [15].

According to James Jeans, the process of mass conversion-its conversion to radiation energy-is merely a process of releasing confined radiation (or what we call photons, particles of electromagnetic energy).

Consider the electron, probably the simplest known mass particle. The most realistic model has it configured as a double looping photon. The self-looping photon is polarized in a manner that causes the peak electric lines of force to be directed radially outward (and by convention these radial force lines are pointing inward for electrons and outward for antielectrons). See the electron model illustrated in Figure 7. As Scottish physicist J. G. Williamson described it, the "model [is that] of a photon confined in periodic boundary conditions of one wavelength. The [toroidal] topology of this object, together with the photon electric field, give rise to a charge of the of the order of $10^{-19}$ Coulomb and a half-integral spin, independent of its size" [16].

So how does the James Jeans idea of "unbottling imprisoned wave-energy" work for that simplest of mass particles, the electron?

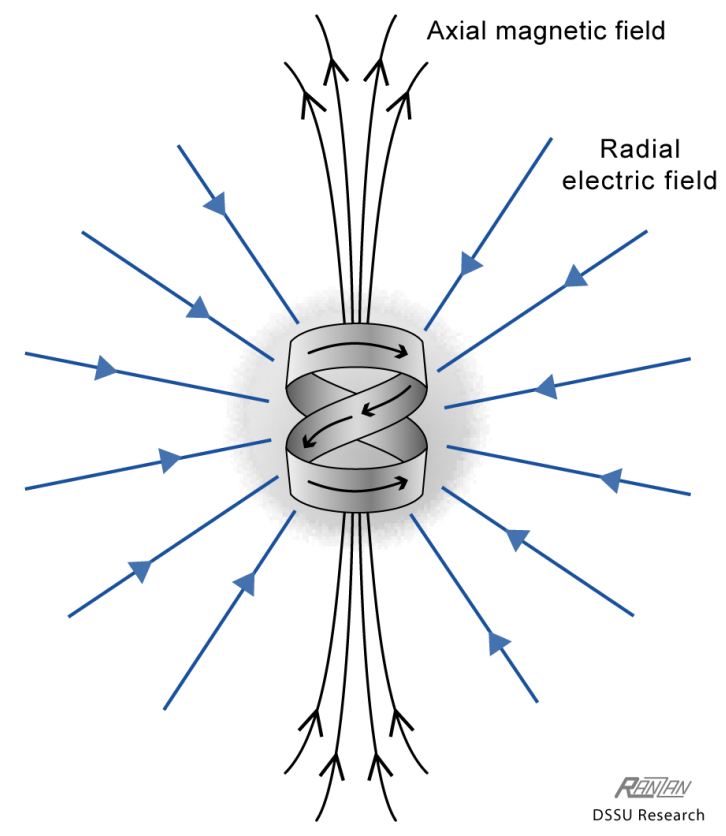

Figure 7. Electron is generated by a two-turn helical path traced out by a photon-a photon confined to a path length of one wavelength. The wave repeatedly reinforces itself with each pass along the double loop. Significantly, the polarization configuration that produced the radial-patterned electric field also produces an axially-directed magnetic field (the electron's dipole) - all in agreement with actual observed electrons. The key lies with the photon's circular polarization: The electric lines of force are oriented inward and perpendicular to the double-loop path represented by the ribbon. And magnetic lines of force are oriented parallel to the ribbon (parallel to the width of the ribbon). 
To better see what is happening, it helps to simplify the double loop of the constituent photon and imagine it stretched into a single loop lying in the $x y$-plane (Figure $8(\mathrm{a})$ ). This does not change the distance the photon propagates; it just simplifies the drawing being used to help explain the mass conversion. Most importantly, since intrinsic motion is being discussed, the coordinate axes in the figure (Figure 8) are stationary with respect to the aether. In other words, the four views show what an imaginary observer, who is embedded in the aether, would "see" when looking at a passing electron.

As a first step, examine the situation for the electron in the rest state (Figure 8(a)). The constituent photon is propagating at lightspeed (as of course it must) while nominally confined within the $x y$-plane. The rotational speed (which here is the same as its tangential speed $v_{\text {tan }}$ ) of the photon is $c$, this means mass (or mass energy) is maximum. The vertical speed of the photon is zero, which means the electron has no kinetic energy, no intrinsic translational motion.

Next, examine the situation for the electron with upward motion (Figure 8(b) and Figure $8(\mathrm{c}))$. The photon's velocity is represented by the tangential vector $\boldsymbol{v}_{\text {tan }}$. Its magnitude is always $c$. But this velocity vector has two components, the rotational $\boldsymbol{v}_{\mathrm{rot}}$ and the vertical $\boldsymbol{v}_{\mathrm{r}}$. The rotational component is associated with the electron's property of mass. The vertical component is associated with the electron's property of kinetic energy. The velocities are related by

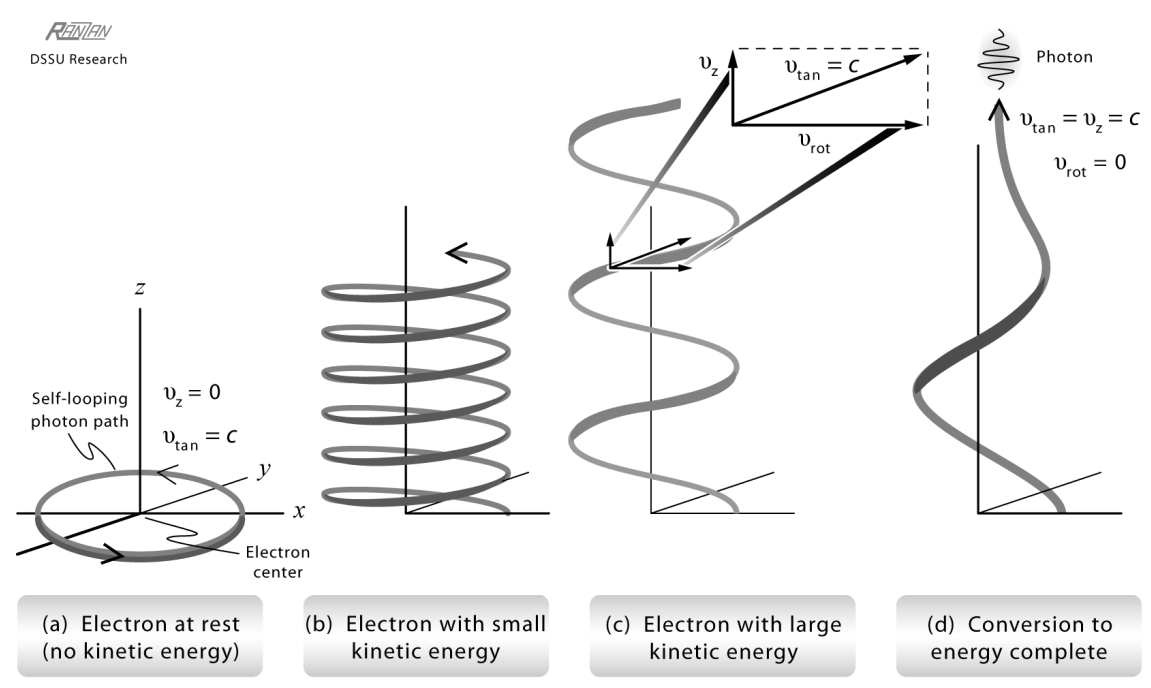

Figure 8. Electron undergoing conversion. Part (a) shows the simplified photon path of a stationary electron. When the electron is in motion, its photon necessarily follows a helical path (b) and (c). The photon's intrinsic-velocity vector can be separated into two orthogonal components: a rotational motion $\boldsymbol{v}_{\text {rot }}$ and a translational motion $\boldsymbol{v}_{\mathrm{z}}$. The velocities of rotation and translation are not independent; they are constrained by the photon's tangential velocity, whose magnitude is constant and always equals lightspeed $c$ (with respect to the aether). The rotational component is associated with the electron's property of mass; and the vertical component is associated with the electron's property of kinetic energy. As the translational velocity increases, the rotational velocity must decrease (compare (b) and (c)). When the rotational component is reduced to zero, when the pitch of the helical path becomes $90^{\circ}$, the photon's propagation becomes linear (d). In other words, in (d), the conversion of the electron's mass into energy is complete. 


$$
v_{\text {photon }}=c=\sqrt{v_{\text {rot }}^{2}+v_{\mathrm{z}}^{2}} \text {. }
$$

Clearly, as the $z$-axis component increases, the rotational component must decrease. In other words, as the kinetic energy of motion increases, the mass energy decreases.

Naturally, at no time can the photon's $\boldsymbol{v}_{\mathrm{z}}$ velocity vector (which is also the electron's translational velocity) exceed the speed of light.

However, under extreme conditions, when the photon's $\boldsymbol{v}_{\mathrm{z}}$ velocity attains lightspeed, the rotational velocity vanishes. And so does the electron's mass. The helical path has transformed to a linear one (Figure $8(\mathrm{~d})$ ); the electron's mass has transformed into pure energy. The trapped electromagnetic wave has been "unbottled".

In the next section, it will be shown how the unbottling speed is actually attainable.

The current section ends with a summary discussion of mass. The property of mass is defined by two essentials. One is the absorption, excitation and vanishment of aether (as discussed in various DSSU research articles available at http://www.cellularuniverse.org/). The other is something best described as electromagnetic wave confinement. This means there must be a non-zero revolving velocity component; that is, the constituent photon (or photons in the case of mass particles more complex than the electron) must revolve, orbit like, about some center. The tighter the confinement (the smaller the loop radius) is, the greater is the mass. Furthermore, the smaller the pitch of the photons helical path is, the greater is the intrinsic mass. Conversely, the greater the helical pitch, the smaller the intrinsic mass. If the helical path is stretched to such an extent that it becomes linear, then the property of mass vanishes - the photon no longer manifests that property. Remarkably the mass has thus faded to zero-and without mass there can be no mass energy. What remains is but the kinetic energy. And so it is for all mass particles, specifically for those subjected to the condition to be detailed below.

What about the opposite process, the fresh formation of mass from the basic energy particle? Mass is formed when free photons are forced into a state of confinement. This occurs in the formation of matter-antimatter paired particles. The classic example is the collision between two gamma photons resulting in the paired formation of an electron and a positron.

The difference, it should be emphasized, between mass and massless particles is utterly simple. Although the massless constituent photon, or photons, always travels at lightspeed (with respect to aether), the mass particle itself must travel at less than lightspeed-this, by virtue of the fact that the orbital center (the center of mass) must travel slower than the looping/orbiting photon(s) as demonstrated in Figure 8.

\section{Astrophysical Situation}

\subsection{Stellar Collapse and the End-State Structure}

Consider a star with a mass equivalent to about 3.4 times that of our Sun. It is at 
the end of its normal lifespan and it has no rotation. Imagine this massive star undergoing a simplified gravitational collapse. No sudden implosion, no rebound ejection, no nova event, and no supernova explosion-just a gradual contraction.

Now, depending on the theory being used, there are two radically different outcomes predicted. One is according to the well-established theory of gravity, which conspicuously lacks a causal mechanism; the other is according to the recently-validated theory of gravity and which does have a causal mechanism [17]. One conforms to the conventional collapse scenario, which, if taken to its logical conclusion, ends in a physical impossibility; the other produces a perfectly natural end-state.

Traditional scenario: The key point in the conventional view is that there is no force in nature that can prevent the star's complete implosion. Given the aggregated quantity of 3.4 Suns, there is simply too much mass. As the star collapses, the mass density rises. It rises until it reaches the ultimate density-nuclear or neutron density. The conventional belief is that stationary maximum-density stars cannot exceed 2.16 solar masses. "With an accuracy of a few percent, the maximum mass of non-rotating neutron stars cannot exceed 2.16 solar masses" [18]. What this means is that if the collapsed object (the neutron-density mass) exceeds 2.16 Suns, then Nature's ultimate push-back, the degeneracy pressure of neutronium matter, is not sufficient to sustain the structure. If the mass exceeds the limit (which it clearly does in the hypothetical example of 3.4 Suns), then there is no known force to prevent total collapse. The collapse follows the scenario shown in Figure 9(a), a scenario with a catastrophic physical breakdown ending in a mathematical entity called a singularity.

Also take note of a critical boundary. Understand that it has two interpretations with radically different implications. Appreciate the difference when comparing the two parts of Figure 9.

The traditional view in a nutshell:

"The view among relativists is that as the mass collapses, it reaches a stage of concentration at which the escape velocity is equal to the speed of light. The associated spherical 'boundary' is, for obvious reason, called an event horizon. The collapse continues, it continues accelerating toward the center of gravity. The collapse ends as a singularity-a point of infinite density. The mass ends up sealed deep within an outer 'boundary.' The singular mass, along with its uncompromising one-way barrier, is called a stellar black hole. It is a most preposterous unnatural end-state" [19].

Natural collapse: The key to understanding the Natural collapse mechanism is in recognizing the convergent flow of aether into mass-any and all mass, as well as any matter that can be converted to mass. Recall, the defining characteristic of mass is its need to continuously absorb/annihilate aether. The very existence of the mass depends on this steady inflow. Now, the nature of fluid dynamics requires that, as the medium converges, the speed of the flow increases. From the two facts just cited, it follows that the maximum flow speed occurs at 


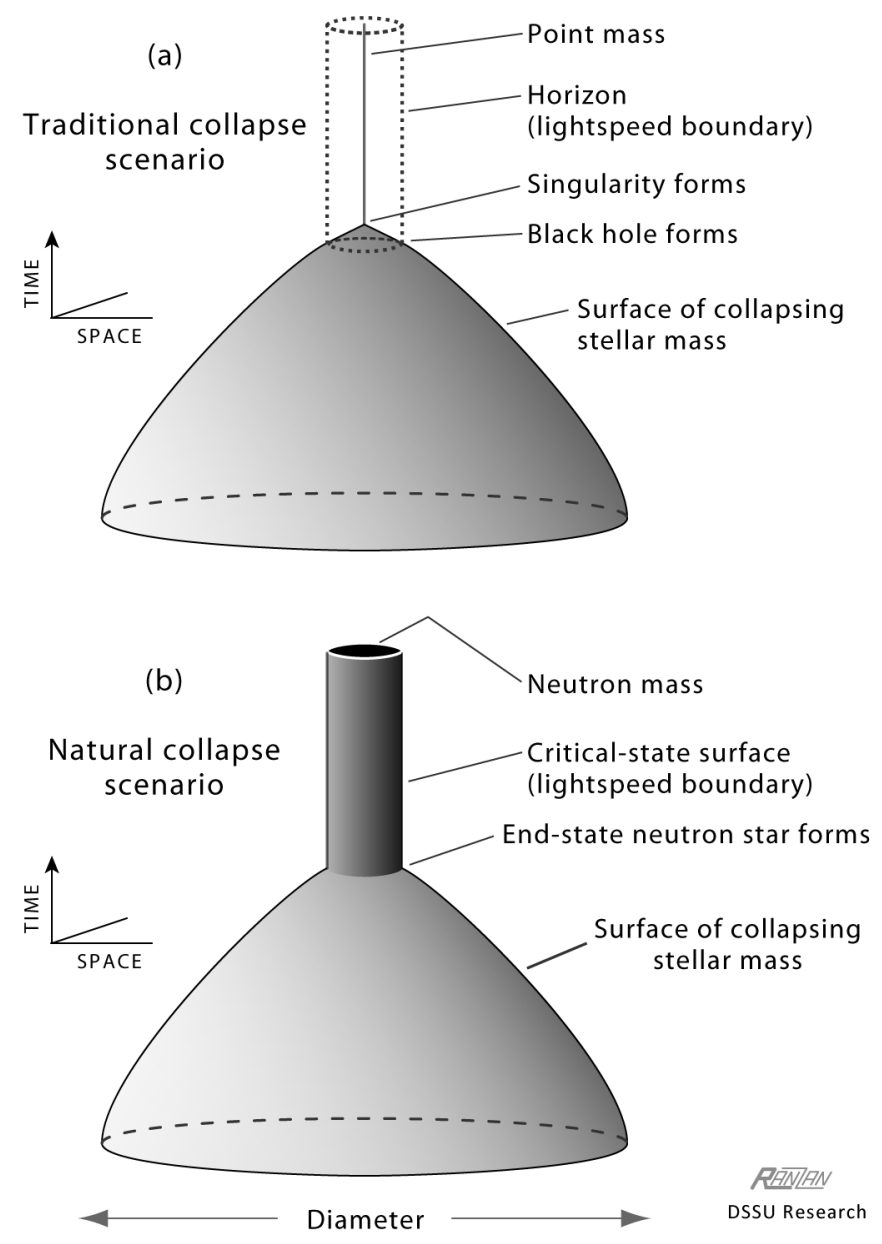

Figure 9. Gravitational collapse scenarios. (a) Schematic of traditional unstoppable gravitational contraction; the result is a singularity-type black hole. Its critical boundary is an event horizon located in free space; (b) Natural end-stage collapse results in a critical-state neutron star. Its critical boundary is a pure-energy layer located on the physical surface.

the surface of the mass structure (of the collapsing stellar structure, in this case). After penetrating the surface, the aether is absorbed/annihilated as it continues its convergent course, but with diminishing speed, towards the center of mass [20].

The important point is this: the inflow is maximal at the surface AND as the area of the surface decreases (due to the contraction of the stellar mass) the flow speed must increase. Therefore, as the structure collapses, and this is still being visualized as a gradual non-violent contraction, it increases until eventually it becomes critical. The aether inflow attains the speed of light (Figure 9(b)).

It is at this stage that the collapse comes to an end. The mass has reached its peak density (neutron density $1.6 \times 10^{18} \mathrm{~kg} / \mathrm{m}^{3}$ ); it has a one-way lightspeed boundary; yet it does not violate the speed rule of special relativity (as will be confirmed shortly). The final product is an end-state neutron star with a radius of 10 kilometers. It simply cannot contract further-even if more mass is added. 
The significant difference: With a singularity-type black hole, the critical boundary is an event horizon located in free space. With a Natural collapse to a Terminal neutron star (another name for the critical-state neutron star), the critical boundary is a pure-energy layer located right on, or at, the physical surface.

The Terminal neutron star has a critical-state surface-a physical one-way boundary.

\subsection{Surface Transitions to Criticality}

The mechanism of conversion of mass to energy resides in the surface region-specifically, in its transition to criticality. The surface region provides the gravitational environment where the transformation can take place.

The familiar electron will again be the focus of the mass-to-energy conversion discussion. This time the electron is kept stationary-motionless at the surface of the gravitating structure-while the aether flows downward onto, and through, the surface. And by making this a thought experiment, we are afforded the luxury of following one and the same electron during selected intervals of the stellar collapse.

The stellar mass is the 3.4-Solar-mass spherical body introduced above. The mass constituting the surface-be it gaseous, plasmic, or solid-is subjected to an aether "wind" in accordance with the following equation, derived in reference [20],

$$
v_{\text {surface.inflow }}=\sqrt{\frac{2 G M_{3.4 \odot}}{R_{\text {surface }}}} .
$$

As the structure contracts to smaller and smaller radius, this equation traces the increase in the flow speed at the surface (Figure 10). The simplest way to interpret the graph is to picture a stop-action sequence; assume that the collapse momentarily stops at each indicated point on the curve, at which point the speed of the aether flow is somehow measured. The collapse, of course, ends when the flow reaches lightspeed-when the radius bottoms out at 10 kilometers.

For example, if the collapse is momentarily halted (easily done, since remember this is a thought experiment) at $R_{\text {surface }}=1000$ kilometers, the boundary-layer mass, including the test electron, will experience an aether flow of one-tenth lightspeed (or $30,000 \mathrm{~km} / \mathrm{s}$ ). When the radius reaches $250 \mathrm{~km}$, the aether flow will be one-fifth the speed of light. Continuing, when the aether speed is checked at radius $40 \mathrm{~km}$, the surface flow will be one-half lightspeed $(150,000 \mathrm{~km} / \mathrm{s})$. And if the collapse is halted when the radius shrinks to $15.7 \mathrm{~km}$, the aether flow will be eight-tenths lightspeed $(240,000 \mathrm{~km} / \mathrm{s})$. Finally, at $10 \mathrm{~km}$ from the center of mass, it all ends; a critical velocity boundary then forms, structural stability is attained, further collapse is precluded. Four of the "stopping points" are indicated in Figure 10.

The electrons embedded in the surface experience the aether flow as a headwind through which they must propagate in order to maintain their surface 


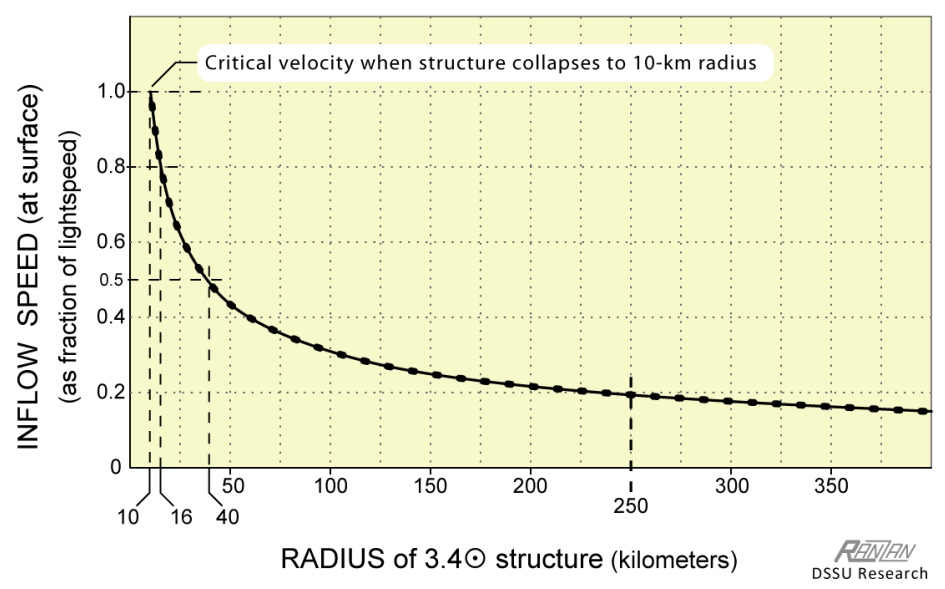

Figure 10. Graph of the aether inflow speed at the surface of a collapsing structure whose mass is equal to 3.4 Suns-in accordance with equation $v_{\text {surface.inflow }}=\left(2 G M_{3.4 \odot} / R_{\text {surface }}\right)^{1 / 2}$. The simplest way to interpret the graph is to hypothetically assume that the collapse momentarily stops at each point while the speed of the aether flow is somehow measured. The collapse necessarily ends at the point where the flow reaches lightspeed-when the radial contraction reaches 10 kilometers.

location. As the collapse progresses and the aether wind increases, the mass attribute of the electrons undergoes its conversion to pure energy. The velocity component responsible for the mass attribute gradually (or incrementally) diminishes to zero, while simultaneously the velocity component responsible for the electrons' intrinsic motion increases to the ultimate speed. This vectorial transition is illustrated in Figure 11.

Consider the purely intuitive perspective. Mass objects or particles cannot exist in (or on) the critical-state surface; this is simply because mass cannot travel at lightspeed. During the collapse process, the structure does have a mass surface; upon collapse completion, that mass no longer exists. But it is not lost instantaneously. The electron did not suddenly go from $9.11 \times 10^{-31}$ kilogram to zero the moment lightspeed is attained. No, the mass loss was a transitional process-the process accompanying the surface transition to criticality (Keep in mind, the stellar collapse sequence often involves long periods of stability between very short periods of contraction). The intuitive answer is found in the principle of mass variance with intrinsic motion.

Nevertheless, there is a mechanism whereby mass does convert instantaneously.

\subsection{Instantaneous Conversion}

This time consider an electron or a chunk of mass freefalling towards a critical-state neutron star. Here is a step-by-step description of what is involved under the "instantaneous" mode of conversion.

1) A critical-state neutron star is a gravitationally collapsed object with a super-dense mass interior surrounded by an energy layer. It is called a Terminal neutron star and has its conceptual roots in DSSU theory (in which it is also 


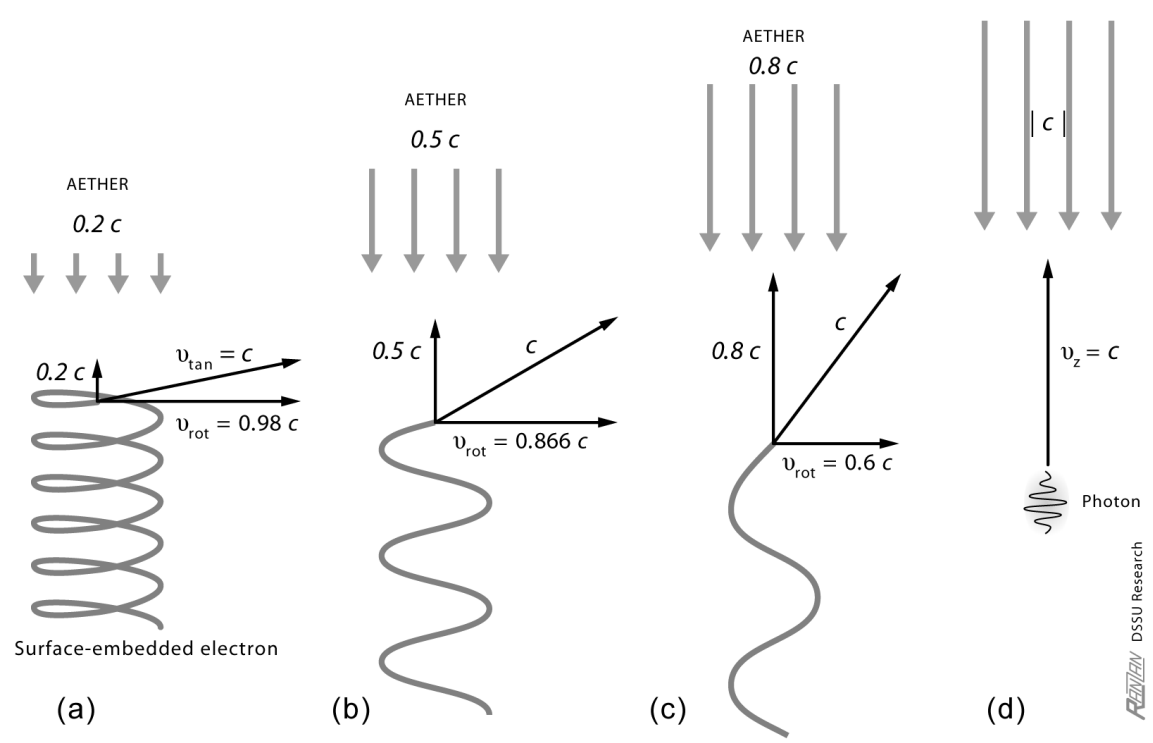

Figure 11. How surface-embedded electrons respond to the velocity of aether inflow at various instances during gravitational collapse (specifically in connection with the collapse plotted in Figure 10). For the electrons to remain stationary at the surface, they must race through the aether "headwind" at (a) $2 / 10^{\text {th }}$ lightspeed; (b) $1 / 2$ lightspeed; and (c) $8 / 10^{\text {th }}$ lightspeed. In (d), the electrons no longer exist but have transformed into their constituent photons. Note, the $\boldsymbol{v}_{\text {rot }}$ component vector determines the electron's intrinsic mass (which varies with the intrinsic speed); and the vertical component vector represents the intrinsic motion.

known as a Superneutron star) [20]. The Terminal star is an extraordinarily strange and stable structure; it does not undergo any further self-collapse.

2) The freefalling mass particles being considered are embedded in the universal space medium; that is, they are flowing WITH the aether. They are on a collision course with the Terminal star.

3) The particles are flowing with the aether and, therefore, are at-rest with respect to it. In other words, their intrinsic energy is entirely mass energy. Their energy-triangle representation would be just a horizontal line (as in Figure 6).

4) The particles, for this example, are not just undergoing ordinary freefall, they are in comoving freefall-comoving with the aether. They are co-accelerating with the aether, continuing all the way up to the speed of light-the speed reached at the very instant of impact with the Terminal star's surface.

5) These particles cannot penetrate to the interior (far too dense) and cannot bounce back (such would violate special relativity); they are effectively trapped in the Terminal star's energy layer. And, it should be mentioned that the energy layer probably has a depth of many centimeters, and may even extend to several meters, as explained in the article, Natural Mechanism for the Generation and Emission of Extreme Energy Particles [21].

6) The very instant the mass particles are stopped, within the energy layer, they convert from $100 \%$ intrinsic mass energy to $100 \%$ photonic energy. In the context of the schematic electron, the self-rotational vector instantaneously becomes the photon's linear propagation vector. 
And so, there are two astrophysical conversion mechanisms-one gradual and one instantaneous. The collapse of high-mass stars (end-state gravitational collapse) provides the environment for gradual mass-to-energy conversion. The existence of critical-state neutron stars makes possible instantaneous conversion.

\subsection{Energy Escape Mechanism}

A natural set of circumstances facilitates the escape of the energy build-up in the surface of the end-state collapsed structure. This structure, the Terminal neutron star, has the following essential features: First, it has a relatively-thin layer of "stationary" high-energy photons (gamma particles) as well as neutrinos (phase-shifted and superpositioned paired photons). Second, a surface that takes the place of a conventional event-horizon boundary, and, exactly like the latter, prevents outbound radiation from escaping. The surface photonic layer rests atop the densest state of mass in existence (of nuclear or supranuclear density). And third, as explained in detail in [21], the structure has a pair of polar "escape holes" in the photonic surface. A couple of other attributes are usually present, but are probably not essential. Notably, there is almost always a magnetic field whose intensity at the poles helps to maintain the integrity of the escape portals. And, of course, a magnetic field is generally associated with rotation. It is a ubiquitous feature in collapsing and collapsed structures; and is invariably present to one degree or another. Although it is not an essential feature for the operation of the emission mechanism, rotation does influence certain characteristics of the external environment (namely jets and any accretion disk that may be present). It should be pointed out that the magnetic field is essential only for the initial formation of the escape portals.

A closer look at the photon and neutrino escape mechanism: The photonic layer is a lightspeed zone whose thickness is delineated on the exterior side by the boundary where the aether inflow first attains lightspeed. This relatively thin layer contains Nature's most energetic electromagnetic waves and undoubtedly holds Nature's densest state of radiation per unit volume. Think of these photons as absolutely saturating their domain-a domain totally inaccessible to investigation from the outside world, so that the enormous density can only be imagined. It is the sheer density of energy in the photon layer that forces the particles toward the edge of the polar portals. Once they reach the polar opening, they escape. They escape at lightspeed; but note, this speed is not with respect to the Terminal star's surface but, rather, with respect to the inflowing aether. Collectively, they form a curtain around the opening-a cylindrical shaft of ultra-high energy blasting into deep space.

The lateral displacement of photons and neutrinos and their escape at the portals never diminishes the surface-layer's energy density. Lost photons can always be replaced by more mass-to-energy conversion as well as by the capture of incoming radiation-the staggeringly abundant stream of cosmic microwave background radiation. An energy amplification process (via the blueshifting of 
the trapped photons) plays a major role in fueling the system. Thus, the streaming of escaping energy is a continuous phenomenon.

Vast energy is continuously generated within, within the surface; and energy is steadily streaming in from without; and energy is sporadically "exploding" onto the surface, as bulk-mass impacts. It all, immediately or eventually, escapes as a ring of radiation-at opposite poles.

\subsection{Quantum Gravity, Black-Hole Energy States and Hawking Radiation}

The section dealing with end-state collapse would not be complete without a brief discussion of mass-to-energy conversion in the context of quantum physics and quantum gravity.

Some new insights, considered to be important among quantum theorists, have recently been obtained within the discipline of quantum gravity. Gained was a deeper understanding of energy states of black holes by treating the Schwarzschild black hole as the nucleus of the "gravitational atom". If a black hole is the nucleus and has excitation states, then the logical question to ask is what should be considered as its corresponding "electrons"?

In the view of astrophysicist and theoretical physicist Christian Corda and his collaborators, by adopting a Bohr-like-atom approach to black-hole quantum physics for the excited black holes, the oscillations (called quasi-normal modes) of the horizon of the Schwarzschild black hole "triggered" by the emission of Hawking quanta and by the potential absorptions of neighboring particles can be considered as the "electrons" of that "gravitational atom". In other words, they interpret the black-hole oscillations as being, in terms of quantum levels of excitation, somewhat similar to the historical semi-classical Bohr model of the structure of a hydrogen atom and its quantum excitation states (with the electron jumping between various energy levels). In a certain sense, the quantum jumps from one level to another triggered by Hawking emissions and corresponding particle absorption represent the energy "shells" of the "gravitational hydrogen atom".

So when a quantum of Hawking radiation is emitted near the event horizon, the "nucleus of the gravitational atom" (i.e. the Schwarzschild black hole) loses mass/energy and the black hole drops to a lower excitation state; this being analogous to the Hydrogen atom having its electron drop to a lower energy state.

Professor Christian Corda's papers detailing this interpretation include [22] [23] [24] [25].

Several assumptions are required: The verity of Hawking radiation; "the unitarity of the black hole quantum evaporation"; the existence of quantum levels of excitation for black holes analogous to the electron's energy states in a Bohr-like atom; the black hole must be nonrotating (the method "works only for the simplest $\mathrm{BH}$, that is the Schwarzschild static $\mathrm{BH}$ "); the "assumption that Schrödinger equations can be used universally for all dynamics in the universe". ... Furthermore, accepted is the fundamental principle of the Schwarz- 
schild paradigm - the density of matter has no upper limit.

Needless to say, the quantum approach is vastly different from the subquantum strategy of DSSU theory. One is based on quantum energy states; the other is based on the dynamics of a subquantum medium.

The quantum-physics mechanism for mass-to-energy conversion and ejection: It is extraordinarily weak; particles are emitted, in Dr Corda's words, "with energy of the order of the Hawking temperature". It is exceedingly protracted; a black hole of the Sun's mass, assuming no additional mass infall, would last $10^{66}$ years! The mechanism requires, as noted above, a number of unsubstantiated assumptions. Significantly, it fails as a source of ultra-high energy particles-not to mention as a driver of astrophysical jets.

The DSSU theory mass-to-energy conversion and ejection: Exploits the subquantum medium, the ethereal essence of the universe; explains the generation of the most energetic particles in all the universe via the most prodigious and intense ejection mechanism; resolves several major astrophysical phenomena; and requires no extraordinary or invalid assumptions. Furthermore, the mechanism works for any Terminal star (critical-state neutron star) whatsoever, rotating or nonrotating, accreting or non-accreting [19] [21].

\section{Implications and Conclusion}

Before discussing several important implications, an important unifying aspect of mass is explained and a quick summary is presented.

The Mechanism applies to ALL mass. Because of the way that it has been defined, all mass is subject to the Conversion Mechanism-all particles and objects that have the attribute of mass are affected by it.

According to DSSU theory all mass particles are composed of confined photons-configurations of self-looping and inter-looping packets of electromagnetic waves. The electron and positron are double looped photons. The muon and tauon are higher-multiple loops. The neutrino is an entwined pair of photons appropriately phase shift so that their individual electric and magnetic fields cancel each other (said to have destructive interference). More complex configurations make up nucleons-the neutron and the proton.

The essential property of mass is some form of photonic self-orbiting, while the defining property of pure energy particles is their free (nominally linear) propagation. When total conversion takes place, the Mechanism frees the photons and allows them to propagate linearly (within the neutron star's surface).

Mass, then, loses its essential property when it is compelled into a lightspeed situation. And that situation only occurs on, and in, the surface energy layer of an end-state neutron star. The Conversion Mechanism is the process that creates that energy layer and thereafter sustains it.

It is worth repeating, the only difference between mass and massless particles is this: Loop-confined propagation versus free-linear propagation. Although the constituent photon or photons always travel at lightspeed, the mass particle itself necessarily travels at less than lightspeed (as was detailed in Figure 8). 


\subsection{Quick Summary}

A quick review. Categorically, mass cannot travel through the space medium at lightspeed. And yet the subject of this article is about what happens when mass is compelled to do so. The resolution to this seeming contradiction presents an opportunity for a quick review.

At the heart of the matter is the explanation of why anything of mass cannot move at lightspeed. By definition, a mass particle consists of at least one self-orbiting photon (as is the case for the electron and the positron). More broadly, mass particles exist as various simple or complex configurations of multiple photons. Also, by definition a photon travels at the speed of light. If the mass particle's constituent photon is to continue to self-orbit, then the photon's forward motion in the direction which it may be forced to travel will necessarily always be less than $c$. The photon's motion must be apportioned between the looping direction and the linear direction-so that the vector sum of the two must be precisely the speed of light.

Clearly then, if mass is compelled to travel at lightspeed, its constituent photon(s) can no longer self-orbit. It no longer meets the requirement of the definition; and it is, therefore, no longer considered to be mass. It simply loses its "mass" property.

A quick reminder about the space medium: It was ignored for decades. But it turns out that the universe's background 3-dimensional space is permeated by aether. However, this is not the $19^{\text {th }}$-century physical aether. Not at all. The modern aether, in its static state, is not any sort of mass-or-energy medium. Rather, it is-when in its static state-nonphysical in conformity with Einstein's medium (as expounded in his 1920 Leyden Lecture [9]). In its dynamic state, it manifests energy.

Quick summary of the two interpretations of the energy triangle: The thing to understand is that the two approaches serve different purposes. The traditional interpretation deals with the "apparent" world-where everything is relative to the observer or the measuring instruments. The new interpretation deals with the intrinsic world-where everything is relative to the universal space medium. The difference is most clearly exemplified by the surface objects/particles of a gravitating body; at one and the same time, they can be apparently stationary AND intrinsically racing through the aether medium.

The traditional understanding employs the measured (or calculated) values of parameters in constructing an apparent-energy triangle; while the universal space medium is not considered. The apparent-energy triangle is applicable to situations where force or energy is or has been applied to a particle or object. The triangle's defining principle is the mass energy remains constant while the total energy may vary (See Figure 12(a)).

The new interpretation employs the intrinsic values of parameters in constructing the intrinsic-energy triangle. It means the universal space medium is respected and, in fact, becomes a key player. The intrinsic-energy triangle is 


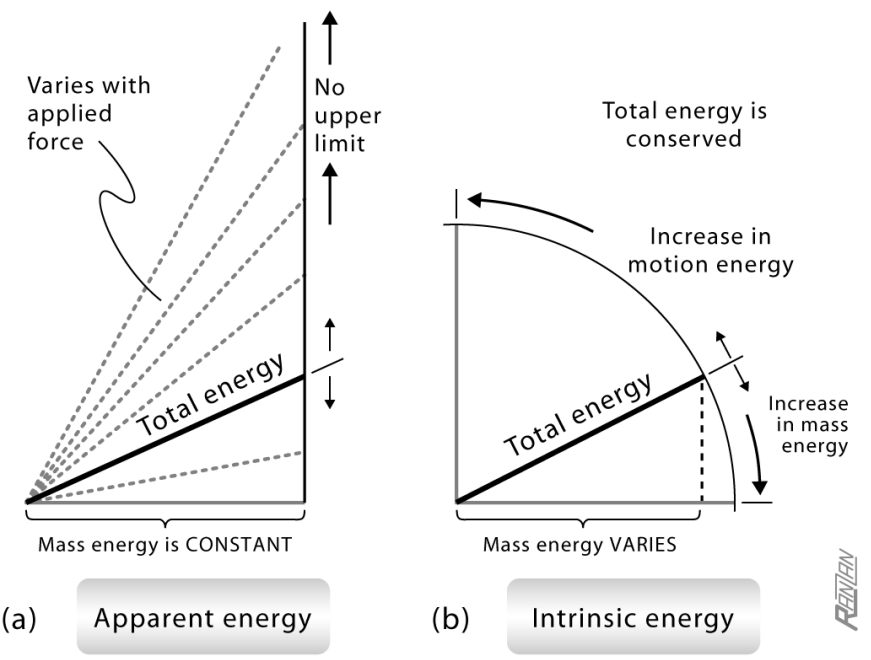

Figure 12. Quick summary of the two interpretations of the energy-relationship triangle. For the apparent-energy triangle, Part (a), the energy of mass remains constant while the total energy (the hypotenuse) varies - it varies with apparent (relative to observer) motion. However, for the intrinsic-energy triangle, Part (b), the energy of mass varies-it varies with intrinsic (relative to aether) motion-while the total energy remains constant. Under the apparent-triangle interpretation, for all the reasons cited earlier, applying energy to a particle will not, not ever, drive it to lightspeed. The essential point is that total conversion can only be modeled with the Part (b) intrinsic triangle.

applicable to gravitational situations-particularly in cases of extreme collapse environments. Here is its defining principle: the total energy remains constant while the mass energy decreases with intrinsic motion (See Figure 12(b)).

Whether working with the apparent or the intrinsic triangle, quantitative conversions are based on the equation $E_{0}=m c^{2}$ (the rest energy).

The most important message revealed in Figure 12 has to do with total conversion. In Part (a), complete conversion is flat-out unattainable, even with the addition of unlimited amounts of energy. Total conversion can only be modeled with the Part (b) intrinsic triangle. In this total transformation of mass-to-energy, no external energy is active (none applied, none involved). Only the gravitational environment is important.

One last point relating to the apparent-versus-intrinsic interpretations. Apparent total energy changes with applied force-with the application of additional energy. However, for the intrinsic interpretation, the total innate energy of a particle does not change-regardless of the gain in kinetic energy from an external force and as long as the confined photon's wavelength does not change. With the gravitational situation, there is no external force (other than the aether flow of the gravity effect, which is not a true force); so, no change in intrinsic energy should be expected. As the length of the hypotenuse reveals (Figure 12(b)), the total innate energy of a mass particle does not change.

\subsection{Implications}

The Conversion Mechanism via gravitational collapse/contraction leads to some 
profound consequences, including the resolution to a number of previously unresolved mysteries.

Nature has two ways for converting mass to energy. There are now two mechanisms for mass-to-energy conversion. Prior to the implications associated with the formation (and mere presence) of Terminal stars, the only known 100\% mass-to-energy conversion mechanism was the one involving the annihilation of particle-antiparticle pairs. The simplest example is the conversion of an electron and a positron into two gamma photons $\left(\mathrm{e}^{-}+\mathrm{e}^{+} \rightarrow \gamma+\gamma\right)$. The inverse reaction has also been observed to occur under appropriate conditions and energy levels; two gamma photons collide to produce an electron and an antielectron. The equation for this particle-antiparticle pair formation is $\gamma+\gamma \rightarrow \mathrm{e}^{-}+\mathrm{e}^{+}$.

Under the particle-interaction mechanism and given the appropriate conditions, nature provides for the mutual convertibility between mass and energy. The reactions can go both ways. However, with the neutron-star mechanism the conversion is, for the most part, a one-way process; the energy of the mass goes into the production of pure energy (gamma photons and neutrinos) which sustains the polar emission beams.

Driver behind astrophysical jets. The Conversion Mechanism provides the primary component of the energy driving astrophysical jets. In accordance with DSSU theory, there are two sources of energy involved. These sources are associated with two distinct mechanisms for generating energy.

One source of energy-the first mechanism-is, of course, the conversion of mass via gravitational collapse and via additional impact, as were discussed earlier. Mass is converted to pure energy, which is then available for powering polar emission jets. But more consequentially, it becomes available for the second mechanism.

The other source of energy comes from what happens within the energy layer enveloping the end-state structure (the structure resulting from the gravitational collapse). The mechanism at work here is best described as an extraordinarily powerful energy amplification process. What is truly profound about this process is that it is, not a mere conversion process, but a veritable energy generating mechanism. For the proof and additional remarkable details, please see the journal-published articles The Nature of Gravitational Collapse and Natural Mechanism for the Generation and Emission of Extreme Energy Particles (Links are provided at http://www.cellularuniverse.org/).

Resolves the black hole mystery. But the most profound implication must surely be the ability of the Conversion Mechanism to provide the resolution to the long-standing problem of stellar black holes. As the journal Nature reported (2014 January), a proper understanding of black holes still does not exist. While it is claimed that quantum theory "enables energy and information to escape from a black hole", a full explanation of the process, would require a theory that successfully merges gravity with the other fundamental forces of nature. But that is a goal that has eluded physicists for nearly a century. "The correct treatment", 
according to physicist Stephen Hawking, "remains a mystery" [26]. But even worse, the singular mass at the heart of any stellar black hole is a far greater mystery-a blatant paradox for conventional cosmology.

When astronomer Subrahmanyan Chandrasekhar (1910-1995) originally developed and promoted the idea in the 1930s of unstoppable gravitational collapse, the influential Sir Arthur Stanley Eddington (1882-1944) dismissed the concept as "absurd" declaring it to be "stellar buffoonery". Einstein weighed in, disavowed the notion, and quite sensibly claimed that some as-yet-undiscovered mechanism would prevent unrestrained collapse.

Enter the Conversion Mechanism. Instead of the absurdity of some infinitely-dense entity (the singularity predicted by simplistic formulations of gravitational collapse), there is now, based on a perfectly natural theory, just an end-state neutron star with a diameter of roughly 20 kilometers.

There simply is no empty region below the lightspeed boundary (the event horizon); there is no "singular" point mass; and there is no paradoxical violation of special relativity.

Moreover, the mechanism of energy generation and ejection, at the fundamental level, can now be clearly understood.

Unexpected implication: After completion of the main body of this article and the write-up of the various, mostly self-evident, implications, there emerged an additional ramification. At first it was just something incidental, then it became an annoyance. However, it could not be ignored. What it revealed was so unexpected, so logically inevitable, and so deeply profound, that a simple assertion of this implication would fail to do it justice and, in the absence of full and proper context, may risk sounding scarcely credible. Decidedly, it demands a proper exposition. It will be the subject of the next DSSU research documentation.

\subsection{The Conceptual Trail}

The conceptual trail started with "unorthodox" relativity and "bottled" energy, followed a natural course through to limitless power, which, in turn, led to stellar astrophysical jets.

The unorthodox approach was to consider the situation relative to the universal space medium. This meant reinterpreting the relativity equations in a way that recognizes that the work required to accelerate a particle from rest to its observed speed is not the same as changing its surroundings, namely its gravitational environment, causing its relative-to-aether motion to increase. Next, the trick was to find a natural way to release the bottled-up energy in mass. The trail led to the pure-energy envelope of the Terminal neutron star in which matter is captured and converted and, in the photonic form, is relentlessly blueshifted to higher energy frequencies. This two-part mechanism provides the limitless power. The astrophysical jets provide the evidence of the validity of the theoretical path thus followed. Where other explanations of the powering of jets fail, the DSSU theory succeeds (see [21]). 
James Jeans, so very long ago, had the right idea. The process of converting mass to energy is merely that of unbottling imprisoned wave-energy and setting it free to travel through space (Interestingly, George FitzGerald, of length-contraction fame, came very close to this same idea decades before Jeans). His insight has led to advances in the understanding of our Universe he could never have foreseen.

In conclusion, it has been shown how to use relativity equations and relativistic motion-in a way that exploits the distinction between apparent and innate levels of reality-to explain mass-to-energy conversion, which, in turn, has led to an explanation of the mechanism driving astrophysical jets.

\section{Conflicts of Interest}

The author declares no conflicts of interest regarding the publication of this paper.

\section{References}

[1] Halliday, D., Resnick, R. and Walker, J. (1997) Fundamentals of Physics Extended. 5th Edition, John Wiley \& Sons, Inc., New York, 974.

[2] Halliday, D., Resnick, R. and Walker, J. (1997) Fundamentals of Physics Extended. 5th Edition, John Wiley \& Sons, Inc., New York, 964.

[3] Halliday, D., Resnick, R. and Walker, J. (1997) Fundamentals of Physics Extended. 5th Edition, John Wiley \& Sons, Inc., New York, 975.

[4] Okun, L.B. (1989) The Concept of Mass. Physics Today, 42, 31. https://doi.org/10.1063/1.881171

[5] Wheeler, J.A. and Taylor, E.F. (1966) Spacetime Physics. W.H. Freeman, San Francisco, 116.

[6] Ranzan, C. (2018) Sachs-Wolfe Effect Disproof-The Fundamental Flaw in the Spectral Analysis of Gravity Wells. International Journal of Astrophysics and Space Science, 6, 1-17. http://dx.doi.org/10.11648/j.ijass.20180601.11

[7] Wang, L.J. (2017) A Critique on Einstein's Mass-Energy Relationship and Heisenberg's Uncertainty Principle. Physics Essays, 30, 75-87. https://doi.org/10.4006/0836-1398-30.1.75

[8] Einstein, A. and Infeld, L. (1938) The Evolution of Physics: The Growth of Ideas from Early Concepts to Relativity and Quanta. Simon and Schuster, New York, 207.

[9] Einstein, A. (1922) Sidelights on Relativity. Translated by: G. B. Jeffery and W. Perret. Methuen \& Co., London; republished unabridged and unaltered (Dover, Mineola, New York, 1983).

[10] Michelson, A.A. and Morley, E.W. (1887) On the Relative Motion of the Earth and the Luminiferous Ether. The American Journal of Science, 34, 333-345. https://doi.org/10.2475/ajs.s3-34.203.333

[11] Miller, D.C. (1933) The Ether-Drift Experiment and the Determination of the Absolute Motion of the Earth. Reviews of Modern Physics, 5, 203-242. https://doi.org/10.1103/revmodphys.5.203

[12] Cahill, R.T. (2006) The Roland De Witte 1991 Experiment (to the Memory of Roland De Witte). Progress in Physics, 2, 60-65. http://www.ptep-online.com

[13] Epstein, H.I. (2009) Could Mass Decrease With Velocity? Physics Essays, 22, 511-514. http://dx.doi.org/10.4006/1.3227028 
[14] Jeans, J.H. (1931) The Mysterious Universe. Cambridge University Press, UK, 51.

[15] Jeans, J.H. (1931) The Mysterious Universe. Cambridge University Press, Cambridge, UK, 51-52. https://doi.org/10.1259/0007-1285-4-43-351

[16] Williamson, J.G. and van der Mark, M.B. (1997) Is the Electron a Photon with Toroidal Topology? Annales de la Fondation Louis de Broglie, 22, 133. https://www.researchgate.net/publication/273418514

[17] Ranzan, C. (2015) Dynamic Steady State Universe Validated by Redshift Theory and Structural Evidence. Physics Essays, 28, 455-473. https://doi.org/10.4006/0836-1398-28.4.455

[18] Goethe-Universität Frankfurt am Main (2018) How Massive Can Neutron Stars Be? Science Daily, 16 January 2018. https://www.sciencedaily.com/releases/2018/01/180116093650.htm

[19] Ranzan, C. (2018) Nature's Supreme Mechanism for Energy Extraction from Nonmaterial Aether. Infinite Energy, 25, March/April 2019. http://www.infinite-energy.com/iemagazine/issue144/index.html

[20] Ranzan, C. (2016) The Nature of Gravitational Collapse. American Journal of Astronomy and Astrophysics, 4, 15-33. http://dx.doi.org/10.11648/j.ajaa.20160402.11

[21] Ranzan, C. (2018) Natural Mechanism for the Generation and Emission of Extreme Energy Particles. Physics Essays, 31, 358-376.

https://doi.org/10.4006/0836-1398-31.3.358

[22] Corda, C. (2015) Precise Model of Hawking Radiation from the Tunnelling Mechanism. Classical and Quantum Gravity, 32, Article ID: 195007. https://doi.org/10.1088/0264-9381/32/19/195007

[23] Corda, C. (2015) Time Dependent Schrödinger Equation for Black Hole Evaporation: No Information Loss. Annals of Physics, 353, 71-82.

https://doi.org/10.1016/j.aop.2014.11.002

[24] Corda, C. (2015) Quasi-Normal Modes: The "Electrons" of Black Holes as "Gravitational Atoms"? Implications for the Black Hole Information Puzzle. Advances in High Energy Physics, 2015, Article ID: 867601. https://arxiv.org/abs/1503.00565 https://doi.org/10.1155/2015/867601

[25] Heydarzade, Y., Hadi, H., Corda, C. and Darabi, F. (2018) Braneworld Black Holes and Entropy Bounds. Physics Letters B, 776, 457-463. https://doi.org/10.1016/j.physletb.2017.11.061

[26] Hawking, S. (2014) There Are No Black Holes. http://www.nature.com/news/stephen-hawking-there-are-no-black-holes-1.14583 


\section{Appendix}

\section{Expressing Total Energy in Terms of Rest Energy and Momentum}

The definition of momentum: $\boldsymbol{p}=m \boldsymbol{v} \gamma$.

The definition of total energy: $E=E_{0}+E_{\text {kin }}=m c^{2} \gamma$.

$$
\begin{aligned}
& \qquad \begin{aligned}
& p^{2}=m^{2} v^{2} \gamma^{2} \\
& \text { Squaring Equation (A1) gives: }=m^{2} v^{2} \frac{1}{\left(1-v^{2} / c^{2}\right)}, \\
&=m^{2} v^{2} \frac{c^{2}}{\left(c^{2}-v^{2}\right)} \\
& p^{2} c^{2}-p^{2} v^{2}=m^{2} c^{2} v^{2} v^{2} m^{2} c^{2}+v^{2} p^{2}=p^{2} c^{2} \\
& \frac{v^{2}}{c^{2}}=\frac{p^{2}}{(m c)^{2}+p^{2}} .
\end{aligned}
\end{aligned}
$$

Square the total energy Equation (A2) and combine with Equation (A3) as follows:

$$
\begin{gathered}
E^{2}=\left(m c^{2}\right)^{2} \gamma^{2}, \\
E^{2}=(m c)^{2} c^{2}\left(\left(1-\frac{v^{2}}{c^{2}}\right)^{-1 / 2}\right)^{2}, \\
E^{2}=(m c)^{2} c^{2}\left(1-\frac{\boldsymbol{p}^{2}}{(m c)^{2}+\boldsymbol{p}^{2}}\right)^{-1}, \\
E^{2}=(m c)^{2} c^{2}\left(\frac{(m c)^{2}}{(m c)^{2}+\boldsymbol{p}^{2}}\right)^{-1}, \\
E^{2}=(m c)^{2} c^{2}\left(\frac{(m c)^{2}+\boldsymbol{p}^{2}}{(m c)^{2}}\right), \\
E^{2}=\left(m c^{2}\right)^{2}+(\boldsymbol{p} c)^{2} .
\end{gathered}
$$

Thus, total energy and rest energy and momentum energy are brought together in one equation. 